\title{
The role of the decision system in sensory and memory experiments using confidence judgments'
}

\author{
DOMINIC W. MASSARO2 \\ UNIVERSITY OF CALIFORNIA, SAN DIEGO
}

\begin{abstract}
This paper uses an artificial "sensory task" (a dice game) to investigate whether it is possible to distinguish between two-state and multistate sensory processes by examining confidence ratings of a group of unpracticed Os. The results indicate that $O$ s use a decision rule that is deterministic so that both operating characteristics and a posteriori probability functions reflect the number of states used by the decision system. Thus, it would seem to be possible to use confidence ratings of $O$ s to evaluate theoretical issues.
\end{abstract}

One major issue in studies of human performance in psychophysics and memory concerns the number of sensory states which the decision system uses to determine responses. The issue has been discussed most strongly in psychophysics (Green \& Swets, 1966; Luce, 1963; Swets, 1961). Usually, the shape of the operating characteristic and the relation of a posteriori probabilities to confidence ratings have been interpreted as evidence for the number of states of the sensory or memory system.

A theory which postulates only two sensory states (i.e., Luce, 1963) can lead to a prediction of a rectilinear operating characteristic which consists of two straight lines and an a posteriori probability function which consists of two discontinuous horizontal lines (Green \& Swets, 1966; Nachmias \& Steinman, 1963; Norman \& Wickelgren, 1965). Theories which postulate a continuous sensory distribution lead to a prediction of curvilinear operating characteristics and smooth, monotonic a posteriori probability functions. Unfortunately, a valid test between two-state and continuous sensory models requires additional assumptions about the decision system. For example, proponents of continuous theories assume that Ss make confidence judgments about their responses by partioning the sensory continuum into regions corresponding to their confidence ratings, an operation that cannot be reliably performed if their are only two sensory states. A good deal of discussion in the literature involves disagreement about how a two-state model might assume that Os give judgments of confidence (see, in chronological order, Watson, Rilling, \& Bourbon, 1964; Larkin, 1965; Watson \& Bourbon, 1965; Broadbent, 1966; Wickelgren, 1968).

Aside from these discussions about how an $\mathrm{O}$ might generate judgments of confidence with only two sensory states, there still exists the important empirical issue of whether or not he does in such a way that the number of underlying sensory states can be disentangled from his responses. The purpose of this paper is to test this possibility by putting the $\mathrm{O}$ in a decision task which is known to have a specific number of possible "sensory" states and then determining whether or not the operating characteristics and a posteriori probabilities reflect the known number of states. This study thus complements the theoretical discussions, regardless of the final resolution of that battle. It can be considered to be an elaboration of Wickelgren's study (1967) in which he showed that Os used a deterministic two-state decision rule in a binary decision making experiment. However, the present study employs a more realistic task and requires confidence judgments from the Os. To make the result even stronger, in this study, a group of unpracticed, naive Os were used (as is more often used in studies of memory) in order to see how averaging across Os affected the results.

The task was the dice game described by Green and Swets (1966, pp. 7-11). Two normal dice are thrown along with one abnormal one. The O's task is to decide which of two values ( 0 or 3) the abnormal die had showing. The total sum of the dice (2 through 15) was announced to the Os, and these numbers constitute the analog of the "sensory" states. Assuming the Os all heard the total correctly, the E (via the dice) has complete control over which sensory state is present at any trial and, by suitable analysis of the data, can select how many states underlie any particular set of data.

\section{Observers \\ METHOD \\ The Os were 23 introductory psychology students fulfilling} a course requirement at the University of Massachusetts.

\section{Procedure}

The dice game was explained to the Os in the following manner:

"I did the following experiment with three dice. Two of the dice were normal with one to six spots on their respective sides. The third die was abnormal in that it had three spots on each of three sides and zero spots on the other three sides. I rolled the dice and observed the total number of spots on the three dice and whether the abnormal die was showing a three or a zero. I did this for a series of trials and recorded the results. Tonight, 1 will give you the total number of spots on the three dice on each trial and your task will be to indicate whether the abnormal die was showing a three or a zero. Also, you will rate your confidence in your decision from 1 (least confident) to 5 (most confident). Are there any questions?"

The Os were given 288 trials without feedback of the "actual" value of the abnormal die followed by 288 trials with feedback. The trials were randomized in blocks of 72 so that the number of times each total was presented to the Os equalled the expected number of times the result would occur.

Table 1

The Frequency of the Possible Totals Given that the Abnormal Die was Showing a " 3 " or " 0 " for a Block of 72 Trials

\begin{tabular}{llc}
\hline Dice Total & \multicolumn{2}{c}{ Frequency given Abnormal Die was } \\
" 0 "
\end{tabular}




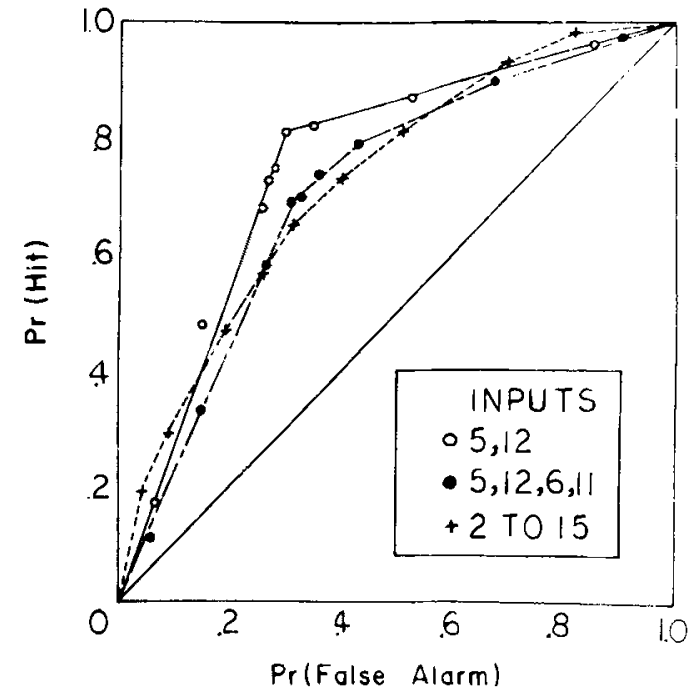

Fig. 1. Operating characteristics pooled over $O$ s and feedback condition as a function of the number of possible inputs.

Table 1 presents the frequency of the possible total given that the abnormal die was showing a " 3 " or a " 0 ." The Os had about $5 \mathrm{sec}$ to make their decision and a 10 -min rest period halfway through the experimental session.

\section{Analysis of Results}

The O's task was to decide whether the abnormal die was showing a 0 or a 3 and to rate his confidence in his decision. In the present study, a hit referred to the $O$ responding " 0 " on a trial when the abnormal die was showing a 0 and a false alarm referred to the responses of " 0 " on a trial when the abnormal die was showing a 3 .

The main concern of this study was the shape of the operating characteristics and a posteriori probability functions analyzed in terms of the number of sensory inputs. Analyzing that subset of trials in which only a 5 or a 12 were given as totals would be analogous to an experiment in which a two-state system was operating. Analyzing all the trials is analogous to an experiment in which a multistate system was operating, since under these conditions there are many (i.e., 14) different sensory inputs that can be discriminated quantitatively by the $O$. Three different partitions of the trials will be considered, with the totals reported to the $\mathrm{O}$ being either 5 or 12 (2 states), $5,6,11$, or 12 ( 4 states), or 2 through 15 (14 states).

\section{RESULTS}

The cumulative hit and false alarm probabilities were computed separately for feedback and no feedback trials. Since these did not differ significantly, all further analyses were pooled over both feedback and no feedback trials. The cumulative hit and false alarm probabilities were computed for different subsets of trials.

Figure 1 presents the operating characteristics analyzed in terms of different subsets of trials. The operating characteristic generated by the cumulative hit and false alarm probabilities from those trials with only two inputs ( 5 and 12 ) indicates that the points are fit nicely by two straight lines intersecting at $(.8$, .3). Figure 1 also shows that with four possible inputs, four straight lines are necessary to fit the observed points. Finally, the operating characteristic constructed from all possible inputs is extremely smooth and requires a curve with many segments.

Figure 2 shows the a posteriori probability functions under the three conditions of Fig. 1. This result agrees with the operating characteristics in Fig. 1 by showing that the number of confidence intervals that Os can reliably employ cannot exceed the number of possible inputs. The figure shows that the a posteriori probability function derived from trials where Os had only two possible inputs is well described by two horizontal line segments. Fig. 2 also shows that the a posteriori probability function becomes more smooth and monotonic as the number of sensory states increases.

It should be noted that the result that the Os were employing a deterministic decision rule is not peculiar to the particular subsets of trials presented in the figures. Many other combinations of input values were analyzed to verify the conclusion that the operating characteristic and the a posteriori probability function reflect the number of states in the system. More specifically, the data in the figures are not due to $d^{\prime}$ value or the number of observations contributing to the curves.

\section{DISCUSSION}

The results indicate the operating characteristics and a posteriori probability functions generated by the rating method were reliable measures indicating the number of states of an underlying system. Hence, the Os in this study were using a decision rule that was deterministic and their responses reflected the underlying system.

In summary, to the extent that this experiment and data analysis were comparable to a real experiment, we can conclude that operating characteristics and a posteriori probability functions generated by the rating method are reliable indices of the underlying sensory or memory system.

\section{REFERENCES}

BROADBENT, D. E. Two-state threshold model and rating scale experiments. Journal of the Acoustical Society of America, 1966, 40, 244-245.

GREEN, D. M., \& SWETS, J. A. Signal detection theory and psychophysics. New York: Wiley, 1966.

LARKIN, W. D. Rating scales in detection experiments. Journal of the Acoustical Society of America, 1965, 37, 748-749.

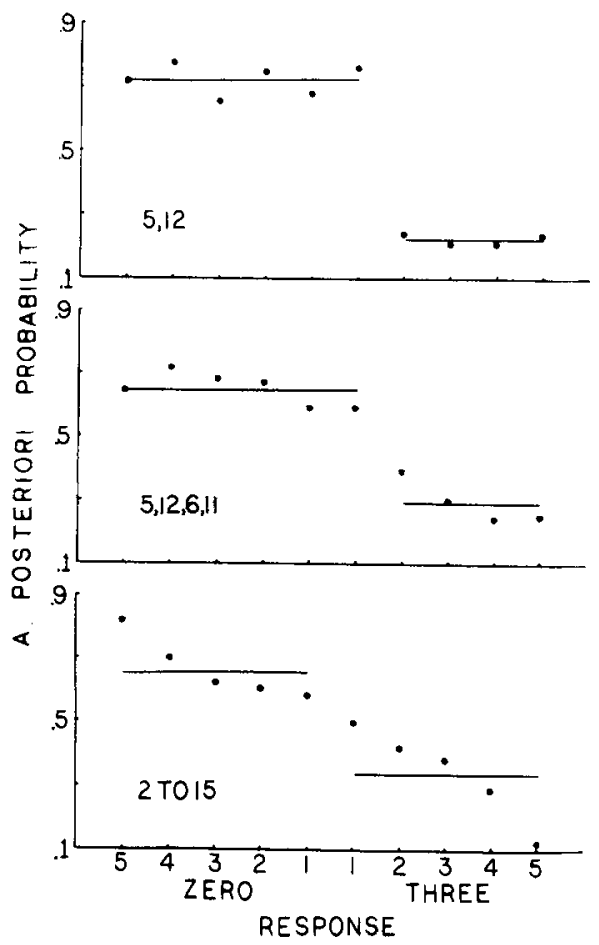

Fig. 2. The a posteriori probabilities pooled over Os and feedback condition as a function of the number of possible inputs. The horizontal lines are usually taken as the predictions of a two state theory. 
LUCE, R. D. A threshold theory for simple detection experiments. Psychological Review, 1963, 70, 61-79.

NACHMIAS, J., \& STEINMAN, R. M. Study of absolute visual detection by the rating-scale method. Journal of the Optical Society of America, $1963,53,1206-1213$

NORMAN, D. A., \& WICKELGREN, W. A. Short-term recognition memory for single digits and pairs of digits. Journal of Experimental Psychology, 1965, 70, 479-489.

SWETS, J. A. Is there a sensory threshold? Science, 1961, 134, 168-177.

WATSON, C. S., \& BOURBON, W. T. Rating scales and two-state threshold models. Journal of the Acoustical Society of America, 1965, $38,667-668$

WATSON, C. S., RILlING, M. E., \& BOURBON, W. T. Receiveroperating characteristics determined by a mechanical analog to the rating scale. Journal of the Acoustical Society of America, 1964, 36, 283-288.
WICKELGREN, W. A. A test of Luce's two-state decision rule. Psychonomic Science, 1967, 9, 91-92.

WICKELGREN, W. A. Testing two-state theories with operating characteristics and a posteriori probabilities. Psychological Bulletin, $1968,69,126-131$.

\section{NOTES}

1. This study was conducted while the author was a National Aeronautics and Space Administration predoctoral fellow at the University of Massachusetts under Grant NSG(T)-137. The author would like to thank Don Norman for his helpful comments on an earlier draft of this paper.

2. Address: Department of Psychology, P. O. Box 109, University of California at San Diego, La Jolla, California 92037.

(Accepted for publication November 27, 1968.) 